\title{
Mountain Papaya Seeds Causing Fecal Impaction in Children
}

Julie Ituku, Mathenge Nduhiu

Nyeri County Referral Hospital, Nyeri, Kenya

Correspondence to: Dr. Julie Ituku. P.O Box 64964-00620 Nairobi, Kenya. Email: juliegituku@gmail.com

\section{Summary}

Stool retention more commonly affects children. It is a clinical finding in which a child resists bowel movements resulting in infrequent defecation, which then becomes larger and more painful. If left unaddressed, it may result in fecal loading or fecal impaction. Fecal impaction is a common gastrointestinal problem and a potential source of morbidity. Prompt identification and treatment therefore minimize the risks of complications. Treatment options include manual disimpaction and proximal or distal

\section{Introduction}

Fecal impaction is a common gastrointestinal disorder and a source of significant patient discomfort with potential for major morbidity especially in the pediatric and elderly population (1). Commonly found in the rectum, it is defined as the inability to evacuate large hard inspissated concreted stool or bezoar lodged in the lower gastrointestinal tract. It is also known as coprostasis or inspissated stool syndrome (1). Stool retention in children is a known precipitating factor. Literature has reported cases due to seeds such as pumpkin, watermelon, pomegranate, sunflower and prickly pear (2). We are however unaware of cases reported due to mountain papaya seeds, particularly in Kenya. We report 3 cases of children who presented to Nyeri County Referral Hospital during the past 2 years, with fecal retention due to undigested mountain papaya seeds with complications necessitating extensive treatment, including manual disimpaction and in one case colostomy. Nyeri County Referral Hospital is the largest of the 7 public hospitals in Nyeri County, which covers an area of $3,337.2 \mathrm{~km}^{2}$, with a population of approximately 720,708 people (3). On average, 204,000 people are seen at the hospital annually, $5 \%$ of who are below the age of 18 years. washout in the absence of complications. We report 3 cases of children who presented in the past 2 years, with stool retention due to undigested mountain papaya seeds.

Key words: Prickly mountain papaya seeds, Stool retention, Fecal impaction, Manual evacuation.

Ann Afr Surg. 2017; 14(2):113-116

DOI:http://dx.doi.org/10.4314/aas.v14i2.13

(C) 2017 Author. This work is licensed under the

Creative Commons Attribution 4.0 International License.

\section{Case 1}

In September 2014, a 9-year-old girl presented with a 3day history of failure to pass stool, which was gradual in onset with normal bowel movements prior. Since then, she reported worsening abdominal discomfort with inability to pass stool even when the urge arose. She was noted to have eaten a mountain papaya (pulp and seeds included) 1 week prior to admission after which she had eaten her usual diet. She reported no history of abdominal distension, abdominal pain, vomiting, anal pain, obstipation or hematochezia. No significant past medical history was reported. At a peripheral facility, several attempts at manual evacuation and enemas were unsuccessful.

Physical examination revealed a sick-looking child who was restless and in obvious pain but had normal vital signs. The abdomen was not distended, moved with respiration, was soft, non-tender and, with normal bowels sounds. There was tenderness on digital rectal examination with hard stool and prickly seeds noted on the blood stained examining finger. The patient was put on a laxative and enema.Two days later, she passed minimal hard stool with pain noted on defecation.No obstructive symptoms had been reported. 
On physical examination, she was still sick looking with normal vital signs. The abdomen was slightly distended, moving with respiration, tense and tender on palpation with normal bowel sounds. Inspection of the anal region revealed an edematous and inflamed anal orifice with visible impacted stool. A decision was made to take the child to theatre for examination under anesthesia (EUA) and manual evacuation. Informed consent was obtained from her guardian.In theatre, the patient was sedated and positioned in lithotomy position. On examination, the anal opening was patulous with surrounding excoriation. There were infected deep anterior and posterior anal tears exposing the internal anal sphincter fibers. The feces were then evacuated digitally and the rectal wall examined and found to be edematous with adherent fibrinous exudate. Intraoperatively, the decision was made to divert stool post evacuation to allow healing. A divided sigmoid colostomy was fashioned and reversed 5 months later. There was a delay in reversal of the stoma due to difficulty finding theatre space for elective procedures in our hospital.

\section{Case 2}

In May 2015, an 8-year-old boy presented with a history of abdominal pain, abdominal distension, vomiting and failure to pass stool for 5 days. He had no significant past medical history. He reported eating mountain papaya with the seeds prior to the onset of symptoms. A soapy enema had been attempted in a peripheral facility unsuccessfully. Physical examination revealed a sick-looking, dehydrated and febrile boy, a distended abdomen that was moving with respiration, tender with bowel sounds present. There was hard stool noted on digital rectal examination without blood on the examining finger. An initial diagnosis of intestinal obstruction secondary to fecal impaction was made and management with broad-spectrum antibiotics, analgesics, enemas, a nasogastric tube and intravenous fluids instituted. Two days later, the patient had not passed stool and a decision was made to do manual disimpaction of the stool under conscious sedation, with the patient in lithotomy position. Prickly mountain papaya seeds were found in the stool during evacuation and a laceration noted at 6 o'clock in the anal mucosa, with an intact internal anal sphincter. He was then given a laxative and analgesic. The patient's symptoms improved minimally and a repeat evacuation was done 3 days later as he was still unable to pass stool, save for the little amount of watery stool he passed soon after manual evacuation. In the repeat evacuation, more seeds were removed. The patient was later discharged on a laxative, analgesic and sitz baths, which resulted in remarkable improvement and relief of symptoms.

\section{Case 3}

In July 2016, 6-year-old girl with no significant past medical history presented with a history of abdominal pain, anal pain and failure to pass stool for 3 days with no vomiting or nausea. She reported eating mountain papaya with the seeds prior to the onset of symptoms. Physical examination revealed a slightly distended abdomen moving with respiration, non-tender with bowel sounds present. There was tenderness on digital rectal examination, hard stool, prickly seeds and blood on the examining finger. Plain supine abdominal radiographs done showed increased right colonic fecal residue. One loop of distended bowel was seen in the mid abdomen. There were however no significant fluid levels and no pneumoperitoneum. Manual evacuation of stool was done under sedation with the patient in lithotomy position and patient given analgesia and a laxative for 5 days post evacuation (as shown in Figure 1). Permission was obtained to allow publication of the images. On follow-up, the patient's symptoms resolved completely.

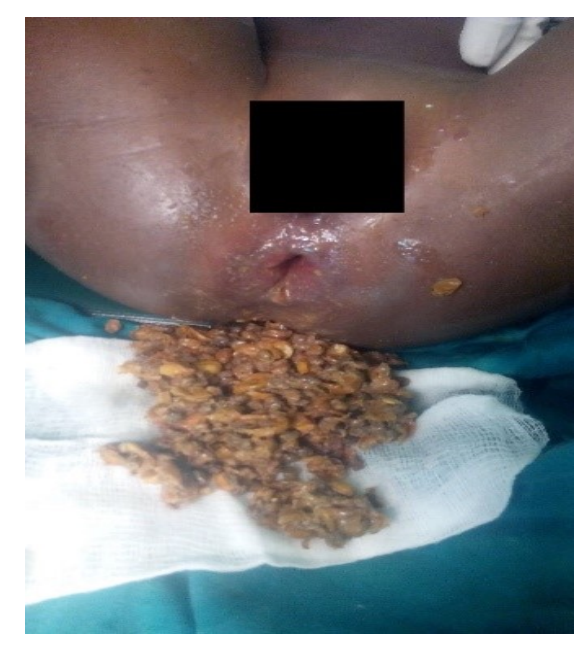

Figure 1: Manually evacuated stool containing seeds

\section{Discussion}

The mountain papaya (Vasconcelleapubescens) is an evergreen, pachycaul shrub or small tree growing to 10 $30 \mathrm{ft}$. It naturally grows in a cool tropical-subtropical 


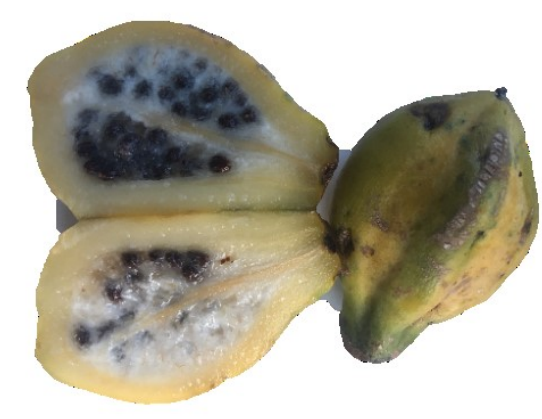

A

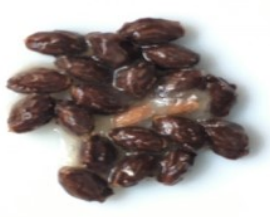

B

Figure 2: (A) Mountain papaya fruit. (B) Seeds of the fruit

climate with annual temperatures between $10-24^{\circ} \mathrm{C}$ and enjoys lots of rainfall (4). It typically grows at altitudes of 4000-8000ft (5). The fruit is $6-15 \mathrm{~cm}$ long and 3-8 $\mathrm{cm}$ broad, with five broad longitudinal ribs from base to apex; it is green, maturing yellow to orange. The fruit pulp is edible, similar to papaya, and is usually cooked as a vegetable. It is also eaten raw (4). In Kenya, it is commonly found in the highland areas (6) like Nyeri County that lies at an altitude of 1500 meters above sea level (7) unlike the Carica papaya, which are warmseason plants and do better in lowland areas. There are unique differences between the mountain papaya fruit and the more common Carica papaya. The flesh of mountain papaya is thinner making about $60 \%$ of the fruit weight (8) unlike the Carica papaya whose pulp comprises $79.5 \%$ of the fruit weight (9). It also has numerous prickly seeds within the central cavity covered by a sweet, transparent, and gelatinous membrane (8) unlike the Carica papaya with a hollow central core, fewer spherical seeds and a bitter, less palatable pulp surrounding the seeds (as shown in Figure $2 \mathrm{~A}$ and $\mathrm{B}$ ). It is for these reasons that we think children consume both the flesh and seeds of the mountain papaya fruit. The entire Carica papaya plant, leaves and roots included, has several known nutritional benefits. The flesh of the fruit has laxative properties while the seeds (ingested once ground) have antibacterial properties (10). It is possible that the mountain papaya shares similar properties however there is little literature about its nutritional benefits.

The 3 cases presented above demonstrate stool retention following the ingestion of mountain papaya seeds. Once evacuated the seeds are prickly and hard, possibly due to the extraction of water as they pass through the digestive system. This is in contrast to their soft nature prior to ingestion. We presume that they cause bowel evacuation problems as a result of reluctance to pass stool due to the pain caused on attempted defecation as the sharp ends irritate and sometimes tear the mucosal lining. Also, possibly once the seeds are dehydrated, they clamp together forming a ball of stool thus requiring a lot of force to expel them. In the second case, the boy developed fecal impaction and required several attempts to expel the hard stool.

The typical presenting symptoms of fecal impaction are similar to those found in intestinal obstruction from any cause, including abdominal pain and distention, nausea, vomiting, and anorexia (1). In all 3 cases, the children presented with failure to pass stool for 3-5 days following ingestion of the fruit. This is fairly unusual in our set up as most patients have high fiber intake. For this reason most children pass stool 1-3 times a day. Abdominal pain, abdominal swelling and vomiting were other reported symptoms. Treatment methods used in various comparable studies included liquid paraffin, warm water enema (11), polyethylene glycol, colonoscopy with roth retrieval nets and baskets (12), manual digital extraction (1) and instrumental extraction (13). In a child with small bowel obstruction due to lentil soup remnants who had previously undergone surgery for acute appendicitis and was being managed conservatively, exploratory laparotomy with an enterotomy to decompress the bowel was necessary due to the deteriorating condition of the child (14). The use of laxatives and enemas had been attempted in these studies as the first line treatment unsuccessfully. In one study, the use of colonoscopy with roth retrieval nets and baskets was done six times with only $60 \%$ removal of the pumpkin seeds. Manual finger fragmentation of the seeds was necessary before a final colonoscopy could be performed to complete the evacuation. Instrumental extraction in addition to 
digital evacuation was successful in a patient in one of the comparable studies.

Fecal impaction may cause mechanical obstruction of the colon. It causes an intraluminal pressure increase of the colon, and therefore ischemic phenomena are produced, which may lead to ulcer and colon perforation resulting in bleeding and peritonitis (15).

A lot of literature is available on fecal impaction due to bezoar formation (11-13); however our cases were due to seed impaction. This is because in all 3 cases, the seeds had been ingested short term and therefore did not form a solid mass of seeds but rather were mixed within the stool.

We believe that there are possibly many children who consume the fruit with the seeds but do not develop fecal impaction. It is possible that those who do have functional constipation, poor dietary fiber intake or it may be the quantity of the seeds consumed that predisposes them to the impaction. This may present an opportunity for further research.

\section{Conclusion}

In children presenting with symptoms of fecal impaction, following ingestion of mountain papaya seeds, manual evacuation of stool should be the immediate method of choice. This is because from the 3 cases reported, conservative management was unsuccessful. We believe that manual evacuation is easy and safe to perform, with minimal invasiveness. It also reduces the progression to intestinal obstruction as well as the risk of complications associated with it.

\section{References}

1. Obokhare I. Fecal Impaction: A Cause for Concern? Clin Colon Rectal Surg. 2012; 25(1):53-8.

2. Arie E, Israel MK, Yechiel S, et al. Fecal Impaction in Children: Report of 53 Cases of Rectal Seed Bezoars. Journal of Pediatric Surgery. 2007;42(6):1114-1117

3. http://www.nyeri.go.ke/wpcontent/uploads/2017/01/ NYERI-COUNTYANNUAL-DEVELOPMENT-PLAN-FY2017.2018.pdf(2-7-2017)

4. http://research.omicsgroup.org/index.php/Mountain papaya (13-3-2017)

5. https://www.therarevegetableseedconsortium.com/s hop-for-seeds/Andean-Mountain-Papaya-Caricapubescens-p58877574(7-3-2017)
6. Asudi GO, Ombwara FK, Rimberia FK, et al. Morphological Diversity of Kenyan Papaya Germplasm. Afr. J.Biotechnol. 2010; 9(51): 875462.

7. Muturi P. A Review of Nyeri County-Kenya Strategic Plan 2013-2017. Int. J. Economics Commerce Manag. 2015; 3(5):1365.

8. Morales AL, Duque C. Aroma Constituents of the Fruit of the Mountain Papaya (Caricapubescens) from Colombia. J. Agric. Food Chem. 1987; 35(4):538-40.

9. Medina J, Gutiérrez GV, García HS. Papaya: Postharvest Operations. Food and Agriculture Organization of the United Nations. 2003: 2. Available: URL: http://www.fao.org/3/a-av012e.pdf accessed 24th Mar2017)

10. Vijay Y, Pradeep KG, Chetan SC, et al. Carica papaya Linn: An Overview. Int. J. Herb. Med. 2014; 2(5):1-8.

11. Jao SW, Wang LT, Wu CC, et al. Removal of a Giant Rectosigmoid Phytobezoar without Laparotomy. Indian J Surg. 2015; 77(Suppl 1):6971.

12. Manne JR, Rangu VM, Motapothula UMR, et al. A Crunching Colon: Rectal Bezoar Caused by Pumpkin Seed Consumption. Clin Med Res. 2012; 10(2):75-77.

13. Minty B, Kelly L. Rectal bezoars in children. CMAJ : Can J. 2010; 182(11):E532.

14. Plataras C, Sardianos N, Vlatakis S, et al. An Unusual Cause of Small Bowel Obstruction in Children: Lentil Soup Bezoar. BMJ Case Rep. 2014; 2014:bcr2013203316.

15. Serrano FB, Barceló LM, Mateos MB, et al. Fecal Impaction: A Systematic Review of its Medical Complications. BMC Geriatr. 2016; 16:4. 